\title{
Joint Involvement in Ochronosis
}

\section{Biehl $\mathbf{C}^{1^{*}}$, Thormann $\mathrm{U}^{1}$, Madera $\mathrm{N}^{2}$ and Heiß $\mathrm{C}^{1}$}

${ }^{1}$ Clinic and Polyclinic for Trauma-, Hand and Restorative Surgery, Justus-Liebig-University Giessen, Germany

${ }^{2}$ Diakonia Hospital, Bad Kreuznach, Germany

\begin{abstract}
Introduction: Ochronosis is a metabolic disorder that is usually associated with the typical brown-black colored urine and retention of phenol complexes in sclera and skin. Kidney and heart are also checked, the disease can also cause damage in these organs. The disease is less associated with degenerative changes in the joints of the limbs and the spine.
\end{abstract}

Methods: We report on the progress of a patient with documented family history on alcaptonuria and joint involvement. In the age of 69 the patient got a total hip and knee arthroplasty. Intraoperatively, signs of massive osteoarthritis beside the clinical picture of brown-black synovia were detected. Histopathology confirmed these findings.

Results: After arthroplasty of the right hip and both knee joints in 2007 and 2008 the patient is symptom-free. Improvement of the serum and urine levels could be achieved by diet. The remaining joints are not treated surgically yet.

Conclusion: In patients with metabolic defects, joints should be checked properly to detect deterioration in an early stage and specifically treated with all available therapies (dietetics, physical therapy, etc.) to delay surgical treatment.

Keywords: Alcaptonuria; Ochronosis; Endoprosthetic replacement; Autosomal recessive defect; Knee replacement; Black cartilage; Fibrosis

\section{Introduction}

Ochronosis is the clinical picture of the metabolic disease alcaptonuria. Alcaptonuria is an autosomal recessive defect of homogentisic dioxygenase (HGO) and has an incidence of 1:200 000. Homogentisic (HGA) is an intermediate in tyrosine and phenylalanine degradation in which it is in the non-hazardous for the body 4 malylacetoacetat di-oxygenated by breaking the ring structure. Ultimately, this is then broken down via intermediates as fumarate and acetoacetate via the citric acid cycle in water and $\mathrm{CO}_{2}$ (Figure 1).

When ochronosis, the toxic metabolite of homogentisic, is enriched in collagen connective tissue and partly excreted in the urine. In the endogenous form of ochronosis the etiology is known (error in homogentisic-acid metabolism) and pathogenesis (homogentisic via urine and accumulation in connective tissue). Therefore, it combines with the endogenous form to a disease.

But there are also acquired forms whose etiology and / or pathogenesis are not or only partially known. Acquired ochronosis may occur after contact with toxic agents such as Hydroxychinin, phenols, benzene and picric acid. Usually there is a local reaction in the sense of dermatitis caused by the topical application (first description in 1912).

The different stages are different:

1. Redness and mild hyperpigmentation

2. hyperpigmentation and 'caviar-like' lesions and eventually

3. Papulo-nodular lesions.

The clinical picture was first described by Rudolf Virchow in 1865 . The condition was named after the yellow (ocher-same) staining of the tissue on microscopic examination. However, the affected tissue structures appear macroscopically bluish-gray due to the Tyndall effect.

In a rare disease, the statements are based on a number of descriptions of individual cases or small groups of patients.

\section{Symptoms and clinical appearance}

Many patients remain initially asymptomatic for years or decades

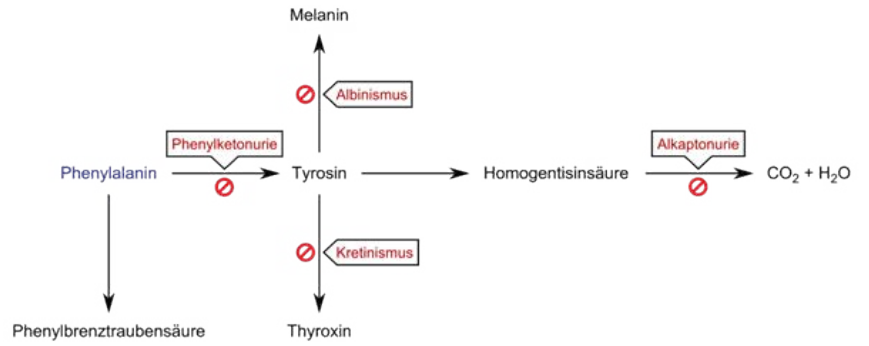

Figure 1: Simplified schematic of tyrosine metabolism disorders*

*Pacelchen: File:Phenylketonurie Schema.svg. Wikipedia.15.05.2009

or show non-specific symptoms in the affected organs, which also occur in other diseases (for example, low-back-pain). Therefore, in a confirmed diagnosis of ochronosis the advice should be cautious and primarily include the recommendation to control the internal organs.

Leading the way of a suspicion of an ochronosis is the bluish/ brownish darkening of urine after short in the air, which is based on the oxidation of the excreted homogentisic in the urine. Also pigmented sclera are hints of an ochronosis. However, the diagnosis of ochronosis is often pronounced in late adulthood, usually when symptoms occur with the involvement of the skeletal system caused by wear of the joints and intraoperatively find further evidence for the disease.

*Corresponding author: Dr. Christoph Biehl Oberarzt, UKGM University, Clinic of Giessen Marburg Clinic and Polyclinic for Accident, Hand and Restorative Surgery-Operational Emergency Room-Rudolf-Buchheim-Str. 7,D-35392, Giessen, Germany, Tel: 0641-985 44 601; Fax: 0641-985 44 609; E-mail: Christoph.biehl@chiru.med.uni-giessen.de

Received September 19, 2016; Accepted October 24, 2016; Published October 29, 2016

Citation: Biehl C, Thormann U, Madera N, Heiß C (2016) Joint Involvement in Ochronosis. Surgery Curr Res 6: 279. doi:10.4172/2161-1076.1000279

Copyright: $\odot 2016$ Biehl C, et al. This is an open-access article distributed under the terms of the Creative Commons Attribution License, which permits unrestricted use, distribution, and reproduction in any medium, provided the original author and source are credited. 
The accumulation of homogentisic in different tissues directs firstly to damage the collagen synthesis, and on the other hand to an accumulation of toxic products with indirect damage to the affected collagenous tissue. Most affected are the para-articular tendon and the hyaline cartilage of the hip, knee, shoulder, the intervertebral discs and the cartilage of the ear and nose.

The most serious consequence for the patient is the involvement of the internal organs such as the vascular system (Aortic valve calcification and of the intima of large vessels) and the kidney with the tendency to nephrolithiasis. At heart and blood vessels, it may subsequently provoke a coronary heart desease and directs to a stroke [1]. The majority of exogenous lesions are found on sun-exposed areas of the body. Typically, the darker skin with brown-gray or blue-black hyperpigmentation.

\section{Diagnosis}

Rapidly and progressive complaints of the joints, which could impress as arthritis or pseudogout, should evoke differential diagnostics of a metabolic disorder. Simultaneously rheumatic diseases and a ochronosis can co-exist, so that the diagnosis of a disease does not automatically exclude the other [2]. Unlike degeneration due to osteoarthritis related wear, the delay between the onset of symptoms and arthroplasty is less. Patients with ochronosis tend to show earlier signs of wear, compared with patients with osteoarthritis. The suspected diagnosis of Alkaptonurie can be debugged with the paper / film chromatography. Blood plasma and urine are suited for diagnosis.

In healthy patients homogentisic is lacking both in blood plasma and urine. Homogentisic plasma levels of more than $6.6 \mathrm{pg} / \mathrm{ml}$ and in the urine of an average of $3.12 \mathrm{mmol} / \mathrm{mmol}$ creatinine are proofing the existence of a Alkaptonurie.

\section{Conservative treatment}

Till nowadays no effective therapy exists to reduce the complications of alcaptonuria. The treatment follows the principles of the conservative treatment of osteoarthritis with anti-inflammatory drugs and physical therapy. In addition, the administration of high doses of ascorbic acid (vitamin C) and dietary restriction of phenylalanine and tyrosine is often recommended. Unfortunately no benefits have been demonstrated in adults.

\section{Surgical treatment}

Due to the rapid and strong deterioration of the joints, the arthroplasty is usually the only possibility of treatment in affected joints (Figure 2a). The postoperative procedure is therefore determined by the changes of collagen tissue in the periarticular structures (tendons, ligaments, etc.).

\section{Case Report}

A 69 year old man comes to the arthroplasty of his knee. General condition are age appropriated and he demonstrates slightly overweight with a BMI of 28.9 (normal 20-25, age-rescaled 24-29) before. The records described a usual range of motion, a polyarthrosis on the large joints (hips, knees, shoulder joints) and a drug treated arterial hypertension, hypercholesterolemia, and a generalized osteoporosis, even without fractures. Only the quickly changing urine in the investigation mug (coke aspect) is remarkable.

When retesting requirements postoperatively the idea of further clarification of the unusual findings towards ochronosis comes on. A determination of homogentisic in urine is not performed preoperatively.

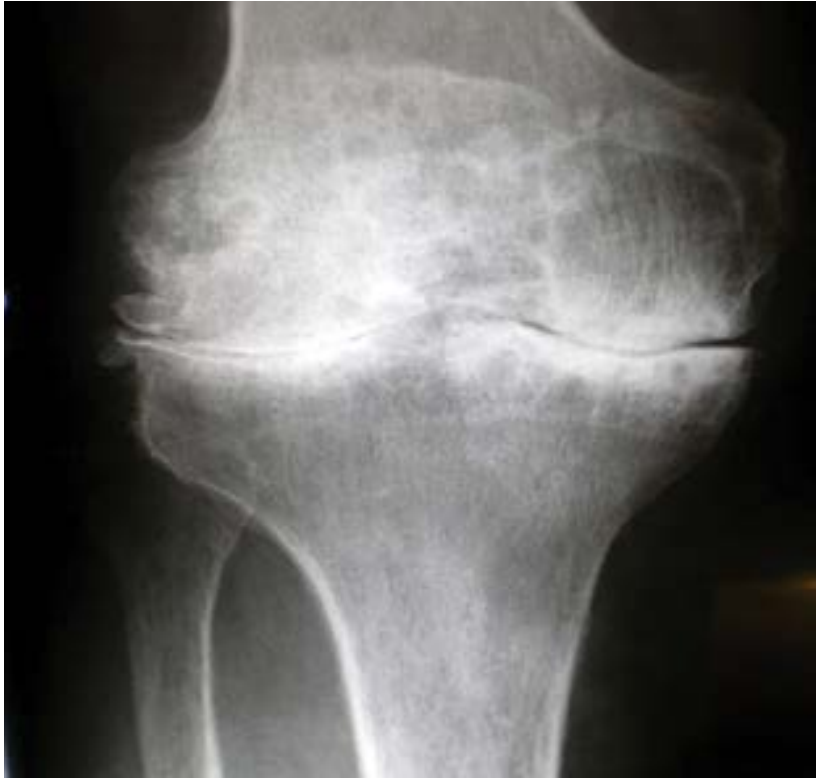

Figure 2a: X-ray of the right knee.

Other signs of ochronosis, as the discoloration of sclera and ear cartilage, cannot be found. The plasma levels of the homogentisic is however determined preoperatively, but is less than $5 \mathrm{pg} / \mathrm{ml}$. Family history towards ochronosis/alkaptonurie is empty. Both parents, siblings and daughter showed no symptoms, she has no allele. The determination of homogentisic in urine is not forced, but other differential diagnoses (methemoglobin, hemochromatosis, etc.) considered. The ferritin in the blood is within normal limits.

\section{Orthopedic Surgeries}

Like many other patients with ochronosis this patient had multiple arthroplastys of the joints [3].

- $\quad 03.2007$ hip prosthesis right

- $\quad 09.2007$ knee prosthesis left

- $\quad 08.2008$ right knee prosthesis

Intraoperatively a total loss of articular cartilage in all compartments at the hip as well as at the knee could be found as a proof. This was associated with a reactive synovitis and osteoarthritis. Preoperative X-ray showed an advanced wear of the articular surfaces with exhaustion of the joint space and small-spotted osteodestructive next to osteoreconstructive processes.

Intraoperatively, the joint surfaces showed a striking black staining of cartilage parts (Figure $2 b$ ) with a mixed picture of subchondral sclerosis and bone defects. Furthermore the joints had large osteophytes, despite a brief clinical symptom progress. Histopathological examination of the fabric gives the pattern of a intertrabecular fibrosis with a low synovitis (score 2) and a brownish discoloration of the cartilage. The first suspected diagnosis of ochronosis was ultimately verified by the histopathological findings.

\section{Spine report}

Intravertebral disc calcifications found on the spine adjacent to small vacuum phenomena and discus calcifications in the sintered intervertebral discs beside a deformity of the spine like a stiffed scoliosis 
Citation: Oberarzt CB, Thormann U, Madera N, Heiß C (2016) Joint Involvement in Ochronosis. Surgery Curr Res 6: 279. doi:10.4172/21611076.1000279

[4]. The advanced degeneration of the spine directs to restriction of movement at all levels with a reduction in Schober's sign [5]. Because of the clinical picture of recurrent inflammatory symptoms a seronegative arthritis was first assumed (Figure 3).

\section{Conclusion}

In arthritis, rapidly progressive disorders of the spine or peripheral joints, wherein the rheumatologic diagnosis (ANA, anti-CCP, HLA-B27, eg) can deliver no suitable values, an accumulation disease should be assumed as a cause and excluded. In diagnosis of ochronosis the level of alcapton in blood and urine together with the pathognomonic urine is conclusive for the disease. If these values seem unremarkable, provides only the exact and also repeated history indications of an exogenous cause, or exposure of the person concerned.

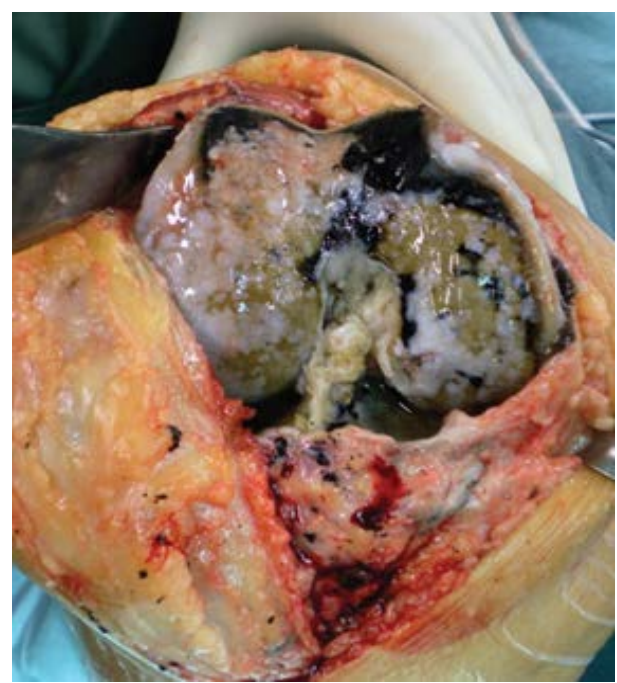

Figure 2b: Intraoperative findings of the right knee.

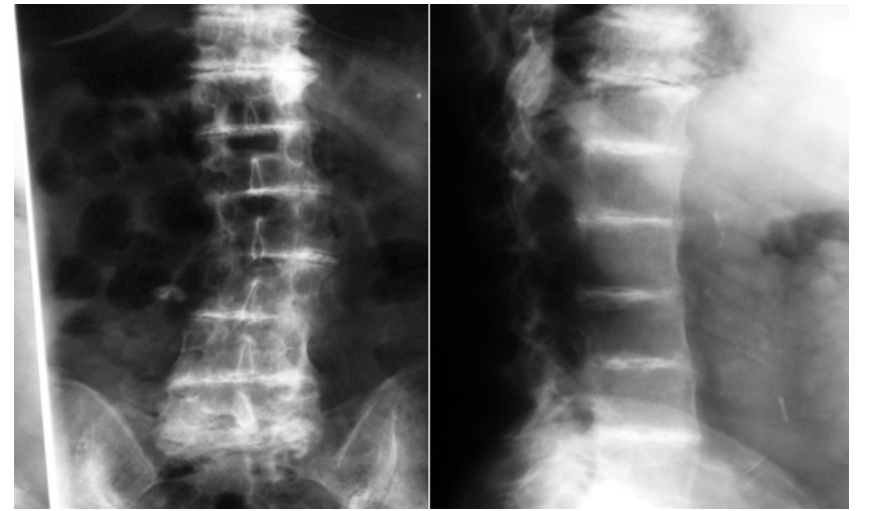

Figure 3: Lumbar spine.

\section{References}

1. Breer S, Amling M, Püschel K, Klein A, Schulz F, et al. (2012) Ochronose. Rechtsmedizin 22: 49-53.

2. Laktasic-Zerjavic N, Curkovic B, Babic-Naglic D, Potocki K, Prutki M, et al (2010) Acute shoulder pain on both sides as the initial symptom of ochronosis. Zeitschrift fur Rheumatologie 69: 443-446.

3. Siavashi B, Zehtab MJ, Pendar E (2009) Ochronosis of hip joint; a case report Cases journal 2: 9337.

4. Dihlmann W (2002) Joints, vertebrae compounds: Clinical Radiology including computed tomography - diagnosis, differential diagnosis. Thieme.

5. Acar MA, Erkocak ÖF, Aydin BK, Altan E, Şenaran H, et al. (2013) Patients with Black Hip and Black Knee Due to Ochronotic Arthropathy: Case Report and Review of Literature. Oman Medical Journal 28: 448-449. 